\title{
LAYER BASED PARTITION FOR MATRIX MULTIPLICATION ON HETEROGENEOUS MESH NETWORKS
}

\author{
Yang Liu \\ Uber Technologies Inc \\ 555 Market Street, San Francisco, CA, USA \\ yangliu89415@gmail.com
}

Junwei Zhang

Uber Technologies Inc

1191 2nd Ave, Seattle, WA 98101

junwei.zhang@stonybrook.edu

\author{
Li Shi \\ Snap Inc \\ Ocean Front Walk, Venice, CA \\ lishi.pub@gmail.com
}

Thomas G. Robertazzi

IEEE fellow

Stony Brook University

100 Nicolls Rd, Stony Brook, NY 11794

thomas.robertazzi@stonybrook.edu

\begin{abstract}
While many approaches have been proposed to analyze the problem of matrix multiplication parallel computing, few of them address the problem on heterogeneous networks. It still remains an open question on heterogeneous networks to find the optimal schedule that balances the load within the heterogeneous processor set while minimizing the communication volume. A great many studies are based on rectangular partition, whereas the optimality of rectangular partition as the basis has not been well justified. In this paper, we propose an alternative approach called layer based partition (LBP), which jointly optimizes the total communication volume and task completion time. We also take network topology into account, by applying LBP on mesh networks. Simulation shows LBP reduces the total communication volume by $81 \%$, while balancing load among all heterogeneous processors in mesh networks.
\end{abstract}

Keywords: Matrix Multiplication, Heterogeneous processing, Load balancing, Communication volume

\section{INTRODUCTION}

Matrix multiplication has been widely performed in a variety of areas. For example, in image processing, a multiplication of projection matrix and coefficient matrix is used to reconstruct the original images from the projections (Zeng 2009). In signal processing, the discrete Fourier transform of a signal is calculated by multiplying the DFT matrix with the signal matrix (Lyons 2010). Many other applications include cryptography, computer graphics, economics, physics, electronics, etc.

On homogeneous networks, the problem of scheduling matrix multiplication load for parallel processing has been extensively studied, such as Canon's (Canon 1969), SUMMA (Geijn and Watts 1994) and Solomonik's 2.5D algorithm (Solomonik and Demmel 2011), etc. However, these approaches generally ignore the heterogeneity of processors and links, as well as network topologies. Thus, on heterogeneous platforms, those 
algorithms fail to apply because they can't guarantee load balance in those scenarios, without considering the heterogeneity of the system. Additional factors need to be taken into account, such as heterogeneous processor speeds and link speeds, network topologies, distributed storage, and etc.

To schedule matrix multiplication on heterogeneous networks, researchers usually consider the following questions.

1. How to allocate the computing load to minimize the total communication volume?

2. How to minimize the task completion time?

To optimize the total communication volume, many previous research works apply rectangular partition on the result matrix (Kalinov 2004)-(Beaumont, Boudet, Rastello, and Robert 2001). Rectangular partition, which adopts the well-known divide and conquer strategy, divides the result matrix into multiple subrectangles, and assigns each sub-rectangle's computing load to different processors respectively. However, approaches based on rectangular partition generally have the following drawback: the best communication volume of rectangular partition may not be globally optimal.

Motivated by this, we proposes another approach called layer based partition (LBP). Rather than assigning one rectangular sub-matrix of the final result matrix to a specific processor to process, our algorithm assigns each processor with one layer. Each layer is of the same shape with the result matrix. We will show that this method guarantees the optimality of the total communication volume.

We further study the problem of scheduling matrix multiplication on heterogeneous processor networks with the goal of minimizing the multiplication completion time. While several approaches have been proposed (Lastovetsky 2007)-(Nagamochi and Abe 2007), none of them addressed problem in the context of a specific network topology, like a heterogeneous mesh. Besides, most previous works consider the problem in the real number domain such that theoretically a processor can get 0.3 rows for instance, which doesn't make sense. Comparatively, we consider the problem in the integer domain which is more applicable in real practice.

\subsection{Our Contributions}

Our main contributions are:

1. We propose layer based partition(LBP) scheme. We show that our scheme is superior to rectangular partition because: it can reach the lower bound of total communication volume.

2. We study the load scheduling problem to minimize task finishing time in heterogenous mesh networks. We also provide a heuristic to reduce time complexity.

\section{RELATED WORK}

Approaches on homogeneous platforms generally assume that all the resources and environment are identical. These approaches include Canon's (Canon 1969), SUMMA (Geijn and Watts 1994), Solomonik's 2.5D algorithm (Solomonik and Demmel 2011). However, as we discussed previously, approaches that are suitable for homogeneous computing aren't necessarily suitable for heterogenous computing.

Approaches on heterogeneous platforms. Efforts have been devoted to analyze matrix multiplication on heterogeneous platforms (Kalinov 2004)-(Beaumont, Dubois, Guermouche, and Lambert 2015). Some researchers try to extend those parallel processing algorithms from homogeneous platforms to heterogeneous platforms (Kalinov 2004)(Quintin, Hasanov, and Lastovetsky 2013)(Ohtaki, Takahashi, Boku, and Sato 2004). 
However, these approaches only optimize communication volume, yet fail to consider whether multiplication completion time gets optimized as well. Therefore, other researchers seek new perspectives, including (Alonso, Reddy, and Lastovetsky 2010)(Malik, Rychkov, Lastovetsky, and Quintin 2014)(Demmel, Eliahu, Fox, Kamil, Lipshitz, Schwartz, and Spillinger 2013)(Beaumont, Dubois, Guermouche, and Lambert 2015). The most utilized partition method in these approaches is rectangular partition. There exists a significant amount of research digging into the problem of rectangular partition on heterogeneous platforms, as discussed below.

Rectangular partition approaches on heterogeneous platforms. Many approaches have been proposed to find the optimal rectangular partition of matrix (Lastovetsky 2007)-(Fuenschuh, Junosza-Szaniawski, and Lonc 2014). However, the optimal partition that minimizes the total communication volume remains an open question. Nagamochi et al. (Nagamochi and Abe 2007) propose a recursive partitioning algorithm that dissects a rectangle into rectangles with specified areas. Ballard et al. (Ballard, Demmel, and Gearhart 2011) study the lower bounds of communication volume, and the difficulty of finding communication optimal rectangular partition. Beaumont et al. (Beaumont, Boudet, Rastello, and Robert 2001) (Beaumont, Dubois, Guermouche, and Lambert 2016) study the complexity to find communication optimal rectangular partition of a specific matrix. Fuenschuh et al. (Fuenschuh, Junosza-Szaniawski, and Lonc 2014) present a polynomial time approximation algorithm that solves a soft rectangle packing problem, and derive an upper bound estimation on its approximation ratio.

In summary, researchers have begun to realize the difficulty in finding the optimal rectangular partition that balance loads while minimize communication volume. In contrast, in layer based partition scheme, we make it very easy to obtain a communication-optimal partition, and show that it reaches the lower bound of total communication volume.

\section{LAYER BASED PARTITION(LBP)}

In this section we propose a new method - the layer based partition(LBP) scheme to tackle matrix multiplication on heterogeneous networks.

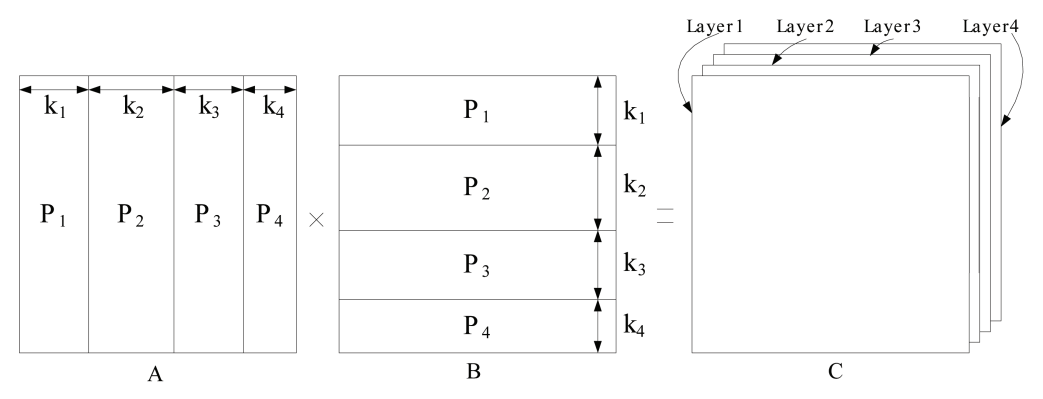

Figure 1: Layer Based Partition Pattern.

\subsection{Scheme Overview}

While the goal remains as conducting two $N * N$ matrices' multiplication, the approach taken by LBP is different from rectangular partition. In LBP, each processor is responsible for calculating one layer of the output matrix. Each layer is of the same dimension of the output matrix, and the output matrix is the aggregation of all layers.

Figure 1 shows an example of this LBP scheme with four processors cooperating on two $N * N$ matrices' multiplication. Processor $P_{1}$ takes matrix $A$ 's leftmost $k_{1}$ columns and $B$ 's upmost $k_{1}$ rows, and then do multiplication. The result is still a $N * N$ matrix, which is actually the 1 st layer of the final output matrix. 
The same method applies to the rest of the processors, with processor $P_{2}$ taking charge of 2nd layer, processor $P_{3}$ taking charge of 3 rd layer, and processor $P_{4}$ taking charge of the last layer. The final output matrix is the sum of these four layers.

\subsection{Assumption}

In this paper, we assume that we don't send back the distributed results on each processor to a centralized locale. The first reason is that a centralized locale may not necessarily be equipped with enough storage or computing power to handle all distributed results. Secondly, we simply don't need to. Thanks to the distributed storage system, we can store those results in a distributed manner. After all, the aggregation of distributed results is yet another addition process, and is much lighter than multiplication processes. We can simply aggregate the distributed results asynchronously, or only when necessary. Therefore, we assume that our task is completed once all the $O\left(N^{3}\right)$ multiplication procedures are done among all processors. In the concept of rectangular partition, that means we consider task as completed once each sub-rectangle result is acquired on each processor. In the concept of our layer based partition, that means we regard task as completed once each layer of the result matrix has been calculated and stored on each processor. In either way, once all the distributed multiplication results are acquired, we mark that as the completion of the task. This is an important assumption for the comparison between layer based partition and rectangular partition in the latter part of this paper. We apply this assumption when calculating the total communication volume and task completion time.

\subsection{Improvements over Rectangular Partition}

The significant improvements of LBP scheme over rectangular partition is that: with the assumption 3.2, LBP generates less communication overhead which is defined as the total data volume sent out by source. As a matter of fact, LBP actually reaches the lower bound of communication volume, as shown below.

In order to ensure an equal base of comparison, we assume in the following part of this paper that, all source nodes do not take part in computation.

Theorem 1. (Layer Based Partition Theorem) LBP generates the minimal total communication volume in conducting two square matrices' multiplication.

To prove the theorem, we firstly present the lower bound of communication volume.

Corollary 2. For all scheduling schemes for two $N * N$ matrices' multiplication, the lower bound of communication volume is $2 N^{2}$, if the source does not take part in processing.

Proof. Because the source doesn't take part in computing, both matrices have to be sent from the source to the computing node. Each matrix contributes a communication volume of $N^{2}$, so two matrices together are $2 N^{2}$. Since each entry of these two matrices has to be sent at least once, the total communication volume is always greater than or equal to $2 N^{2}$.

Back to Theorem 1. Suppose there are $p$ processors, therefore according to LBP, the task is divided into $p$ layers. Processor $p_{1}$ takes matrix $A$ 's leftmost $k_{1}$ columns and $B$ 's upmost $k_{1}$ rows, and thus the communication volume to transfer the necessary processing data from source to processor $p_{1}$ is $N * k_{1}+N * k_{1}=2 N k_{1}$. Similarly, the communication volume of processor $p_{2}$ is $2 N k_{2}$, processor $p_{3}$ is $2 N k_{3}$, ...etc. The total communication volume is $C_{L B P}=\sum_{i=1}^{p} 2 N k_{i}=2 N \sum_{i=1}^{p} k_{i}=2 N^{2}$ 
As shown above, the communication volume of layer based partition scheme reaches the lower bound. Therefore, layer based partition scheme is communication-optimal.

Corollary 3. Rectangular partition consumes a total communication volume greater than the lower bound.

Proof. In rectangular partition, suppose the output matrix is of size $N * N$, and each sub-rectangle's height is $h_{i}$, width is $w_{i}$, and area is $s_{i}$. The total communication volume according to (Beaumont, Boudet, Rastello, and Robert 2001) is: $C_{R E C}=\sum_{i=1}^{p}\left(h_{i}+w_{i}\right) * N$. Because $h_{i}+v_{i} \geq 2 \sqrt{s_{i}}$, hence we have: $C_{R E C} \geq$ $2 N * \sum_{i=1}^{p} \sqrt{s_{i}}$. In the meanwhile, we have the sum of each sub-rectangle: $\sum_{i=1}^{p}\left(\sqrt{s_{i}}\right)^{2}=N^{2}$. Since each $s_{i}$ is positive and $p>1$, this stands: $\sum_{i=1}^{p}\left(\sqrt{s_{i}}\right)^{2}<\left(\sum_{i=1}^{p} \sqrt{s_{i}}\right)^{2}$. Combined with previous equation, we get: $\sum_{i=1}^{p} \sqrt{s_{i}}>N$.

Take this result back to the definition of $C_{R E C}$, we get $C_{R E C}>2 N^{2}$. Thus the total communication volume of $\operatorname{LBP}\left(C_{L B P}\right)$ is less than rectangular partition $\left(C_{R E C}\right)$.

\subsection{Memory Constraints}

The memory limit can be a tight constraint for layer based partition, when the size of the matrix gets enormously large and exceeds memory. For that case, we need to hold the matrices in hard disks, and batch-load the memory and do processing. We can load the memory of each processor to its limit, do the multiplication and write the results to disk. After that, we continue to load the next batch until completing all data sets. This approach is doable with the fast development of hardware like SSD and etc. But for other matrices with size smaller than memory, LBP is still convenient and bears substantial advantages.

\section{LBP ON HETEROGENEOUS MESH}

Table 1: Table of the Variables for Chapter 4.

\begin{tabular}{r|l}
\hline Variables & Meaning \\
\hline$T_{s}(i)$ & The start time of the $i$ th node in the network \\
$T_{f}(i)$ & The finish time of the $i$ th node in the network \\
$k_{i}$ & The number of columns or rows of the multiplier matrix that are assigned to $i$ th node \\
$\phi(i, j)$ & The volume of load transmitted from node $i$ to node $j$ \\
\hline
\end{tabular}

Table 2: Table of the Constants for Chapter 4.

\begin{tabular}{r|l|r|l}
\hline Constants & Meaning & Constants & Meaning \\
\hline$G(V, E)$ & The mesh network with fixed topology & $p$ & The size of the matrices \\
$S$ & The set of source nodes & The number of nodes in this mesh \\
$\tau(i, j)$ & specifies the position relationship & $T_{c m}$ & Cominverse of the link speed \\
& of two nodes $i$ and $j$ in the network. & $w(i)$ & The inverse of the computing speed \\
& $\tau(i, j)=1$ if $i$ is in a position that should & $T_{c p}$ & Computing intensity constant \\
& transmit to $j$, and $\tau(i, j)=0$ if otherwise & & \\
$D_{i}$ & specifies the storage size of node $i$ & &
\end{tabular}

In the following section, we discuss the details of applying the LBP scheme on heterogeneous mesh networks. We focus on analyzing LBP's load balancing strategy, namely, how to schedule load distribution among different processors in the network so that the task completion time is minimized. We formulate the load balancing problem on heterogeneous mesh as an optimization problem, called Minimize Finishing Time in Layer Based Partition(MFT-LBP) problem. Again, as we mentioned in the introduction part, we mark it 
as the completion of the task once all the distributed multiplication results are acquired on each processor. The definition of variables and constants are listed in the following Table 1 and Table 2.

\subsection{MFT-LBP In Mesh}

In this subsection we focus on the MFT-LBP problem in mesh networks. We denote the mesh network as a graph $G(V, E)$. The graph contains $p$ nodes which forms a dimension of $X * Y$, where $p=X * Y$.

Within the scope of this paper, we assume that we're using data transfer pattern as 'parallel communication and consecutive start'. 'Parallel communication' allows each node to talk to its multiple neighbors at the same time. 'Consecutive start' means that each node has to wait until it receives its whole share of data before it can start computing. We present the MFT-LBP problem in the following.

The objective of this problem is to minimize the task completion time, which is defined as the maximum finishing time of the nodes in the mesh network: Minimize $: \max _{i \in G(V, E)}\left\{T_{f}(i)\right\}$. This objective function contains maximum form of formulas, which usually makes optimization problem harder to solve directly. In order to make it easier, we conduct an equivalent transforming as follows. We introduce one additional unknown variable $T_{f}$, and a set of constraints $T_{f} \geq T_{f}(i), \forall i \in G(V, E)$, which ensure $T_{f}$ to be no earlier than any node's finishing time $T_{f}(i)$. Then we transform the objective function to Minimize $: T_{f}$.

\section{MFT-LBP}

Variables: $\left\{k_{i}\right\},\left\{T_{s}(i)\right\},\left\{T_{f}(i)\right\},\{\phi(i, j)\}, T_{f}$

\section{Objective:}

\section{Constraint:}

$$
\begin{gathered}
T_{s}(i)=0, \forall i \in S, \\
T_{S}(i) \geq \tau(j, i)\left[T_{s}(j)+\phi(j, i) \cdot z(j, i) T_{c m}\right], \forall i \notin S, \\
T_{f}(i)=T_{s}(i)+k_{i} N^{2} w(i) T_{c p}, \forall i \in G(V, E), \\
2 N^{2}-\sum_{j \in G(V, E)} \phi(i, j)=0, \forall i \in S, \\
\sum_{j \in G(V, E)} \phi(j, i)-\sum_{j^{\prime} \in G(V, E)} \phi\left(i, j^{\prime}\right)=2 k_{i} N, \forall i \notin S, \\
\phi(i, j) \geq 0, \forall \tau(i, j)=1, \\
\phi(i, j)=0, \forall \tau(i, j)=0, \\
k_{i} \in \mathbb{Z}_{+}, \forall i \in G(V, E), \\
k_{i}=0, \forall i \in S, \\
2 k_{i} N+N^{2} \leq D_{i}, \forall i \notin S, \\
\sum_{i=1}^{p} k_{i}=N, \\
T_{f} \geq T_{f}(i), \forall i \in G(V, E) .
\end{gathered}
$$

\section{Remarks:}


- Constraint (3) specifies that the start time of those non-source nodes should be the maximum time that they finish receiving all loads from their adjacent neighbors.

- $\tau(i, j)$ is a constant once the mesh network is determined and fixed.

- Constraint (4) defines each node's finishing time. According to LBP, each node's computing load is the total number of multiplication it conducts $k_{i} * N^{2}$.

- Constraint (7) - (8) list different values of $\phi(i, j)$ in different cases.

- The $k_{i}$ in constraint (9) is the number of columns taken by each node, and should be integers.

- Constraint (11) shows that for each node, its storage size should at least be able to store the load from two multiplier matrices $\left(2 k_{i} N\right)$, and the result matrix $\left(N^{2}\right)$.

- Constraint (12) is the normalization constraint. The number of columns taken by each node should sum up to be the side length of the multiplier matrix.

This optimization problem looks very much like a linear programming problem, if without one constraint. As constraint (9) shows, $\left\{k_{i}\right\}$ are positive integers, which make MFT-LBP problem a Mixed Integer Nonlinear Programming problem. To solve it, we propose an algorithm called Phased Minimization of Finish Time with Layer Based Partition(PMFT-LBP).

\subsection{PMFT-LBP}

PMFT-LBP: The PMFT-LBP algorithm contains the following three phases. In Phase I, it relaxes integers $\left\{k_{i}\right\}$ to real numbers and solves the relaxed linear programming problem. In Phase II, based on the optimal real number solution obtained in Phase I, PMFT-LBP determines a feasible integer solution for the original PMFT-LBP problem, which is close to the optimal real number solution. In Phase III, starting from the feasible integer solution obtained in Phase II, PMFT-LBP conducts a "neighbor search" and seeks for local optimal feasible integer solution.

Phase I. In this phase, the PMFT-LBP algorithm relaxes condition (9) to be a positive real number

$$
k_{i} \geq 0, \forall i \in G(V, E) .
$$

and solves a relaxed version of the MFT-LBP problem, called MFT-LBP-relax.

With this relaxation, all constraints and objective function are either linear equality or linear inequality. Hence, MFT-LBP is a LP problem and can be solved to get the optimal real number solution $Q^{*}=\left\{\left\{k_{i}\right\}\right.$, $\left.\left\{T_{s}(i)\right\},\left\{T_{f}(i)\right\},\{\phi(i, j)\}, T_{f}\right\}$.

Phase II. In this phase, PMFT-LBP calls an algorithm called finds an integer feasible solution(FIFS) based on the optimal real number solution $Q^{*}$ obtained from phase I. We firstly round off each $k_{i}$ to its closest integer. The intuition here is that if the real number optimal solution assigns the larger portion of a column to one processor, then assigning that processor with the entire column will have a higher chance to get "closer" to optimality. It is vice versa that if the processor is assigned with the smaller portion of a column, we shall remove its share of this column.

However, the rounding off process alone may not guarantee a feasible integer solution, because it may result in the sum of all $k_{i}$ fail to equal the multiplier matrix's side length $N$, constraint (12). To resolve this problem, we conduct a subtle adjustment. For cases in which the sum is greater than $N$, meaning that there exists duplicate assignments, we shall reduce work load from some processors. Since the processor with the longest finishing time is the bottleneck affecting the overall finishing time, it has the highest priority to reduce its share of loads. On the contrary, for cases in which the sum is less than $N$, meaning that some rows/columns haven't been assigned to any processor, processor currently with the shortest running time has the highest priority to take up the responsibility. 
We don't make the adjustment to one processor all at once. Instead, we conduct the adjustment iteratively. Every iteration we only adjust one row/column, then we update each processor's $\left\{T_{s}(i)\right\},\left\{T_{f}(i)\right\},\{\phi(i, j)\}$ to determine the processor to conduct adjustment on for the next round of iteration. The processor with the longest processing time currently will be the one to remove a row/column from in the next round of iteration, and the processor with the shortest processing time will be the one to take the extra load in the next iteration.

When the sum of all $k_{i}$ equals $N$, we finally find an integer feasible solution $\left\{k_{i}^{\prime}\right\},\left\{T_{s}^{\prime}(i)\right\},\left\{T_{f}^{\prime}(i)\right\},\left\{\phi^{\prime}(i, j)\right\}$.

Phase III. In this phase, PMFT-LBP conducts a so-called "Neighbor Search" process to optimize the feasible solution obtained in phase II. The reason why this feasible solution still need optimization is that the "Rounding Off" procedure in phase II might bring about bias and take our feasible solution "away" from optimal. Therefore, a further optimization is necessary.

The "Neighbor Search" runs in iterations. For the first iteration, it starts from the feasible integer schedule $\left\{k_{i}^{\prime}\right\}=\left\{k_{1}^{\prime}, k_{2}^{\prime}, \ldots k_{a}^{\prime}, \ldots, k_{b}^{\prime}, \ldots k_{p}^{\prime}\right\}$ obtained in phase II, and compares the current overall finishing time with its neighbors'. $\left\{k_{i}^{\prime \prime}\right\}=\left\{k_{1}^{\prime \prime}, k_{2}^{\prime \prime}, \ldots k_{a}^{\prime \prime}, \ldots, k_{b}^{\prime \prime}, \ldots k_{p}^{\prime \prime}\right\}$ is defined as one of $\left\{k_{i}^{\prime}\right\}$ 's neighbors if all $k_{i}^{\prime \prime}=k_{i}^{\prime}$ except for only two dimensions $k_{a}^{\prime \prime}$ and $k_{b}^{\prime \prime}$, where $k_{a}^{\prime \prime}=k_{a}^{\prime}-1$ and $k_{b}^{\prime \prime}=k_{b}^{\prime}+1$, such that $\sum_{i=1}^{p} k_{i}^{\prime \prime}=\sum_{i=1}^{p} k_{i}^{\prime}=N$. If one neighbor $\left\{k_{i}^{\prime \prime}\right\}$ offers shorter overall finishing time than $\left\{k_{i}\right\}^{\prime}$ and the rest of its neighbors, we change load schedule from $\left\{k_{i}^{\prime}\right\}$ to $\left\{k_{i}^{\prime \prime}\right\}$. Then, "Neighbor Search" will use $\left\{k_{i}^{\prime \prime}\right\}$ as a new start point and begins a new iteration. If at some stage, no further optimization can be made towards minimizing overall finishing time, which means the schedule at that stage $\left\{k_{i}^{*}\right\}$ is a local minimal point that has the shortest overall finishing time among all of its neighbors, iteration stops and we say we find our optimal schedule.

\subsection{MFT-LBP-heuristic}

Considering the time complexity of PMFT-LBP algorithm with so many LP-based updates are involved, we propose a heuristic called MFT-LBP-heuristic. This heuristic calculates a good feasible integer solution that is close to the optimal solution with a time complexity which is substantially reduced from PMFT$L B P$. The basic idea is that whether one single row/column should be assigned to one processor or another simply doesn't bring about much improvement of the result. However, simplifying these steps reduces time complexity substantially.

Based on this idea, MFT-LBP-heuristic only keeps phase I the same with PMFT-LBP algorithm. In phase II, after obtaining the optimal real-number schedule $\left\{k_{i}\right\}$, the heuristic rounds off each $k_{i}$ to get $\left\{k_{i}^{\prime}\right\}$ slightly differently. The difference is, if the sum of $\left\{k_{i}^{\prime}\right\}$ doesn't equal $N$, the heuristic sorts all processors in ascending order of their finishing time $\left\{T_{f}(i)^{\prime}\right\}$, which forms an array $\operatorname{Arr}[p]$. If the sum of $\left\{k_{i}^{\prime}\right\}$ is less than $N$, then from the first element in $\operatorname{Arr}[p]$, the heuristic keeps adding 1 to each processor's $k_{i}^{\prime}$ until $\sum_{i}\left\{k_{i}^{\prime}\right\}=N$. Otherwise if the sum of $\left\{k_{i}^{\prime}\right\}$ is greater than $N$, the heuristic starts from the last element of $\operatorname{Arr}[p]$ and minus 1 from each processor's $k_{i}^{\prime}$ one by one until $\sum_{i}\left\{k_{i}^{\prime}\right\}=N$.

In phase III, PMFT-LBP searches each neighbor of current schedule $\left\{k_{i}^{\prime}\right\}$. The time complexity of searching process is $O\left(p^{2}\right)$, where $p$ is the number of processors. Once $p$ is big, the scalability of the algorithm is poor. In order to speed up the local search process, we apply the concept of 'gradient descent' here. At each iteration, we only look at the neighbor $\left\{k_{i}^{\prime \prime}\right\}$ that has the highest chance to decrease the overall finishing time from current schedule $\left\{k_{i}^{\prime}\right\}$. We know that $\left\{k_{i}^{\prime \prime}\right\}$ differentiate from $\left\{k_{i}^{\prime}\right\}$ by just two dimensions $k_{a}^{\prime \prime}=k_{a}^{\prime}-1$ and $k_{b}^{\prime \prime}=k_{b}^{\prime}+1$. If $k_{a}^{\prime}$ is the schedule of the processor that currently takes the longest processing time, while $k_{b}^{\prime}$ is the schedule from the processor that takes the shortest, $\left\{k_{i}^{\prime \prime}\right\}$ then stands the highest the chance to be the neighbor that has shortest overall finishing time. We compare $\left\{k_{i}^{\prime \prime}\right\}$ 's $T_{f}$ with $\left\{k_{i}^{\prime}\right\}$ 's. If $\left\{k_{i}^{\prime \prime}\right\}$ 's is shorter, we use $\left\{k_{i}^{\prime \prime}\right\}$ in the next iteration. Otherwise, since even $\left\{k_{i}^{\prime \prime}\right\}$ cannot further decrease the overall finishing time, the other neighbors probably cannot either. Therefore we take $\left\{k_{i}^{\prime}\right\}$ as the optimal schedule. 
This heuristic only solves LP problems twice. The result of this may sacrifice the overall finishing time a little bit, but reduce the algorithm's time complexity substantially by reducing a lot of time-consuming LP iterative updating processes. As will be seen in next section, the performance of our heuristic is extremely close, and even in cases equal to PMFT-LBP both in terms of communication volume and finish time.

\section{PERFORMANCE EVALUATION}

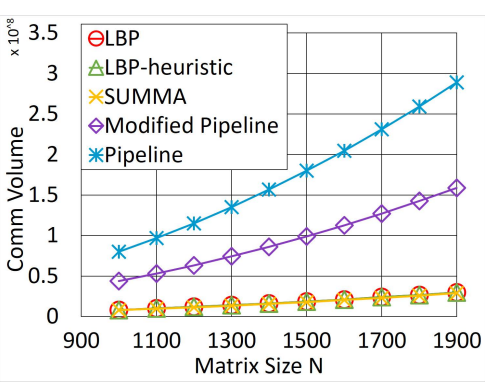

(a) $5 * 5$ Mesh

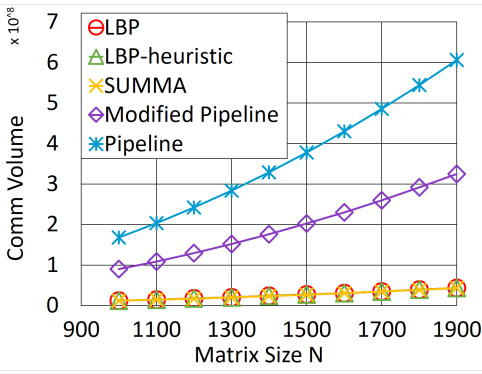

(b) $7 * 7$ Mesh

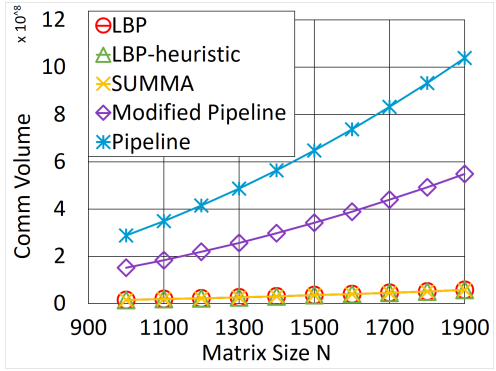

(c) $9 * 9$ Mesh

Figure 2: Communication volume comparison with increasing matrix size and network dimension.

In this subsection, we study how the layer based partition scheme performs in mesh networks. In each single run of the simulation, we randomly generate a heterogeneous mesh network, and two square matrices conducting multiplication. Then our LBP algorithm and the other comparing algorithms are called to schedule the matrix multiplication load on the given mesh network.

Mesh Network. Each link speed and processor speed are independently generated. The unit processing time $w T c p$ of the processors is uniformly distributed in the range of $(0.0005,0.0008)$, while the unit transmission time $z T \mathrm{~cm}$ of the links is uniformly distributed in the range of $(0.0002,0.0005)$. In our simulation, we use use three square meshes, which are of dimension $5 * 5,7 * 7$ and $9 * 9$.

Matrices. The matrices we analyze are large scale dense matrices. In our simulation, we randomly generate matrices with their side length $N$ ranging from 1000 to 2000.

When analyzing the performance of each algorithm over matrix size, each data point in our simulation is an average of 10 independent experiments over 10 independently different mesh network.

\subsection{Comparison Algorithms}

Modified SUMMA. SUMMA (Geijn and Watts 1994) is the most widely applied parallel matrix multiplication scheme on homogeneous grid. We modify this algorithm to apply on heterogeneous mesh network, called Modified SUMMA. In Modified SUMMA there are multiple sources, and each processor in the mesh takes one block of matrix data. So when evaluating the performance of SUMMA, we divide the matrix data into blocks and store it on corresponding processor.

Pipeline. The Pipeline algorithm is a brute-force scheduling method. Starting from the source, each node forwards the entire copy of data along the grids to each of its neighbor in the mesh network. Duplicate copies may be sent to one node, however, it only keep the first received one. Once that node finishes receiving its first copy, it starts processing the data while forwarding.

Modified Pipeline. Tan et al. (Tan 2013) propose an improved pipeline broadcast scheme for distributed matrix multiplication. We apply the idea to heterogeneous mesh network. 


\subsection{Evaluation Result on Mesh}

Overall Communication Volume. Figure 2 displays the overall communication volume when conducting matrix multiplication of two $N * N$ matrices in (a) $5 * 5$ mesh, (b) $7 * 7$ mesh, and (c) $9 * 9$ mesh respectively. The simulation result reveals that while all algorithms's overall communication volume goes up as matrix size increases, SUMMA, LBP and LBP-heuristic generate almost the same smallest overall communication volume, which is $81 \%$ smaller than that of Modified Pipeline and $90 \%$ smaller than that of Pipeline. SUMMA is well-known to be communication-optimal on homogeneous mesh network. Though applying SUMMA on heterogeneous mesh may affect its task finishing time due to the change of processor speed and link speed, its data transmission pattern won't be affected. So SUMMA is still communication-optimal on heterogeneous mesh. LBP and LBP-heuristic generates almost the same communication volume as SUMMA, consequently, implies that LBP and LBP-heuristic are at least close to communication optimal.

Task Finishing Time. Figure 3 shows the task finishing time of each algorithm on (a) $5 * 5$ mesh, (b) $7 * 7$ mesh, and (c) $9 * 9$ mesh. Generally, LBP generates the smallest task finishing time than the rest of algorithms. LBP-heuristic gives a task finishing time that is slightly longer than that of LBP, which are $0.03 \%$ more in $5 * 5$ mesh, $0.08 \%$ more in $7 * 7$ mesh, and $0.18 \%$ more in $9 * 9$ mesh, respectively. This tiny difference can entirely be ignored. SUMMA, since it can no longer reach load balance with link speed and processor speed vary, its task finishing time are, respectively, 56.4\% more in $5 * 5$ mesh, $52.9 \%$ more in $7 * 7$ mesh, and $46.7 \%$ more in $9 * 9$ mesh, than that of LBP. Moreover, Modified Pipeline are respectively $66.7 \%$ more in $5 * 5$ mesh, $87.2 \%$ more in $7 * 7$ mesh, and $121.1 \%$ more in $9 * 9$ mesh. Pipeline are respectively $73.4 \%$ more in 5*5 mesh, $114 \%$ more in $7 * 7$ mesh, and $185 \%$ more in $9 * 9$ mesh. All in all, LBP and LBP-heuristic present the best performance in terms of task finishing time.

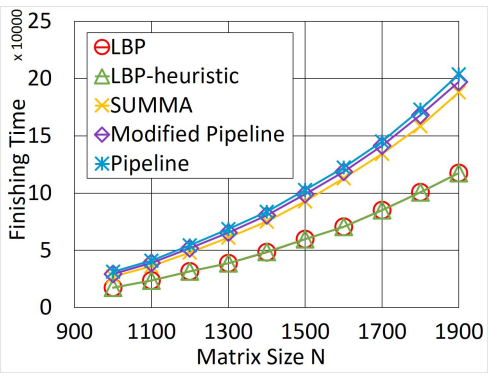

(a) $5 * 5$ Mesh

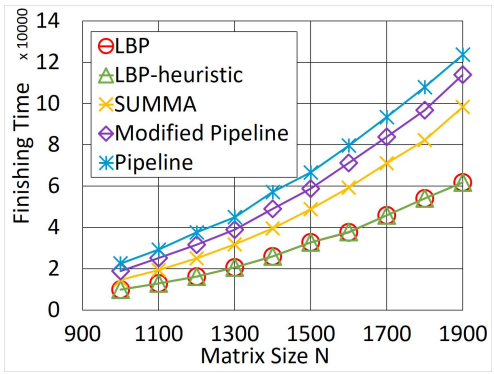

(b) $7 * 7 \mathrm{Mesh}$

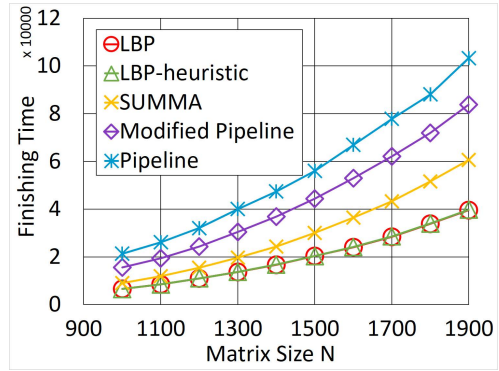

(c) $9 * 9$ Mesh

Figure 3: Finishing time comparison with increasing matrix size and network dimension.

Total Number of Iterations to Solve LP. As mentioned previously, we use the simplex algorithm to solve LP in our LBP and LBP-heuristic algorithm. Each time solving the LP costs a certain number of iterations by the simplex algorithm. And according to our algorithm, we may re-solve LP a couple of times due to 1.find real number solution 2. find integer solution 3. local search, etc. Therefore, the total number of iterations to solve LP is a good indication of the efficiency of our algorithms. Figure 4 counts the average total number of iterations in solving LP by LBP and LBP-heuristic on $5 * 5$ mesh, $7 * 7$ mesh, $9 * 9$ mesh. Each point is an average of 10 identical independent experiments. We have the following observations: 1 . The solid lines vary dramatically due to the uncertain number of times to re-solve LP by LBP, while the dashed lines are comparatively stable. 2 . The total number iterations show no correlation with respect to matrix size, a good evidence indicating that both algorithms are suitable for large scale matrix scheduling. 3. The total number of iterations does show positive correlation with respect to mesh size. 4. For the same mesh size, dashed lines are generally far below solid line, which indicates that LBP-heuristic generally requires much less total number of iterations to find a solution than LBP. In other words, LBP-heuristic is more efficient. 


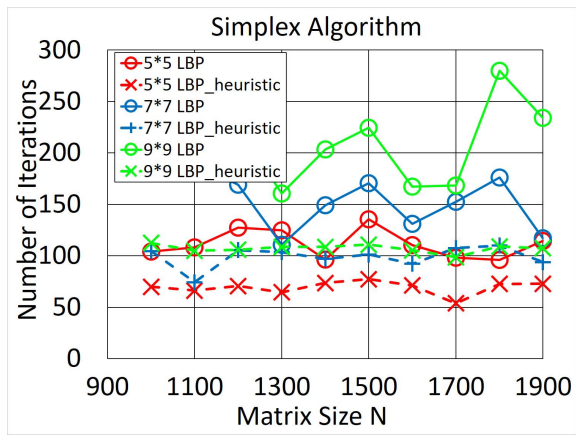

Figure 4: Number of iterations by the simplex method in solving LP.

Summary. Both algorithms outperform the other heterogeneous mesh scheduling algorithms in terms of communication volume and task completion time. Besides, LBP-heuristic is significantly more efficient than LBP while maintaining almost equally good performance, which makes it widely applicable.

\section{CONCLUSION}

In this paper, we focus on the problem of scheduling matrix multiplication on heterogeneous networks. We present a novel scheduling method: layer based partition. We demonstrate that layer based partition scheme generates a total communication volume that is optimal, smaller than that of rectangular partition. In the following part, we study how to minimize task finishing time using LBP on heterogeneous mesh network. We formulate the problem as a Mix Integer Programming problem, which we provide a 3-Phase algorithm. A heuristic algorithm is also proposed to reduce the time complexity.

\section{REFERENCES}

Alonso, P., R. Reddy, and A. Lastovetsky. 2010, Feb. "Experimental Study of Six Different Implementations of Parallel Matrix Multiplication on Heterogeneous Computational Clusters of Multicore Processors". In Parallel, Distributed and Network-Based Processing (PDP), 2010 18th Euromicro International Conference on, pp. 263-270, IEEE.

Ballard, G., J. Demmel, and A. Gearhart. 2011, Feb. "Communication Bounds for Heterogeneous Architectures". In 23rd ACM Symposium on Parallelism in Algorithms and Architectures, SPAA 2011, ACM.

Beaumont, O., V. Boudet, F. Rastello, and Y. Robert. 2001. "Matrix Multiplication on Heterogeneous Platforms". IEEE Transactions on Parallel and Distribute Systems vol. 12, pp. 1033-1051.

Beaumont, O., L. Dubois, A. Guermouche, and T. Lambert. 2015, Oct. "Comparison of Static and Runtime Resource Allocation Strategies for Matrix Multiplication". In Computer Architecture and High Performance Computing (SBAC-PAD), 2015 27th International Symposium on, pp. 170-177, IEEE.

Beaumont, O., L. Dubois, A. Guermouche, and T. Lambert. 2016, May. "A New Approximation Algorithm for Matrix Partitioning in Presence of Strongly Heterogeneous Processors". In Parallel and Distributed Processing Symposium, 2016 IEEE International, pp. 474-483, IEEE.

Canon, L. 1969. "A cellular computer to implement the kalman filter algorithm". In Ph.D. dissertation of Montana State University.

Demmel, J., D. Eliahu, A. Fox, S. Kamil, B. Lipshitz, O. Schwartz, and O. Spillinger. 2013. "Communication-Optimal Parallel Recursive Rectangular Matrix Multiplication". In Proceedings of the 2013 IEEE 27th International Symposium on Parallel and Distributed, IPDPS '13, pp. 261-272, IEEE Computer Society. 
Fuenschuh, A., K. Junosza-Szaniawski, and Z. Lonc. 2014. "Exact and approximation algorithms for a soft rectangle packing problem". Optimization vol. 63, pp. 1637-1663.

Geijn, R., and J. Watts. 1994. "SUMMA: Scalable Universal Matrix Multiplication Algorithm”. Concurrency - Practice and Experience vol. 9, pp. 255-274.

Kalinov, A. 2004, Jul. "Scalability Analysis of Matrix-matrix Multiplication on Heterogeneous Clusters". In Parallel and Distributed Computing, International Symposium on, ISDPC '04, pp. 303-309, IEEE Computer Society.

Lastovetsky, A. 2007. "On Grid-based Matrix Partitioning for Heterogeneous Processors". In Parallel and Distributed Computing Sixth International Symposium on, ISPDC '07, pp. 51-58, IEEE.

Lyons, R. 2010. Understanding digital signal processing. Prentice Hall.

Malik, T., V. Rychkov, A. Lastovetsky, and J. Quintin. 2014, May. "Topology-aware Optimization of Communications for Parallel Matrix Multiplication on Hierarchical Heterogeneous HPC Platforms". In Parallel and Distributed Processing Symposium Workshops (IPDPSW), 2014 IEEE International, pp. 3947, IEEE.

Nagamochi, H., and Y. Abe. 2007. "An approximation algorithm for dissecting a rectangle into rectangles with specified areas". Discrete Applied Mathematics vol. 155, pp. 523-537.

Ohtaki, Y., D. Takahashi, T. Boku, and M. Sato. 2004, Apr. "Parallel Implementation of Strassen's Matrix Multiplication Algorithm for Heterogeneous Clusters". In Parallel and Distributed Processing Symposium, 2004. Proceedings. 18th International, IEEE.

Quintin, J., K. Hasanov, and A. Lastovetsky. 2013, Jun. "Hierarchical Parallel Matrix Multiplication on Large-Scale Distributed Memory Platforms". In IEEE International Conference on Parallel Processing, ICPP' 13 , pp. 754-762, IEEE.

Solomonik, E., and J. Demmel. 2011. "Communication-optimal Parallel 2.5 D Matrix Multiplication and LU factorization Algorithms”. In Euro-Par 2011 Parallel Processing, pp. 90-109, Springer.

Tan, L. 2013, Sep. "Improving performance and energy efficiency of matrix multiplication via pipeline broadcast". In Cluster Computing (CLUSTER), 2013 IEEE International Conference on, IEEE.

Zeng, G. 2009. “Medical Image Reconstruction A Conceptual Tutorial”. Springer.

\section{AUTHOR BIOGRAPHIES}

YANG LIU received his $\mathrm{PhD}$ degree from Stony Brook University. He is now working at Uber Technologies. His research interests are in the area of distributed/parallel computing, task scheduling and resource allocation, high performance computing, and load balancing algorithms. His email address is yangliu89415@gmail.com.

LI SHI received his PhD degree from Stony Brook University. He is now working at Snap Inc. His research interests include task scheduling and resource allocation in data centers, cloud computing, data center network, software defined network, etc. His email address is lishi.pub@gmail.com.

JUNWEI ZHANG received his PhD degree from Stony Brook University. He is now working at Uber Technologies. His research interests include parallel computing optimization, computational geometry and applied machine learning. His email address is junwei.zhang@stonybrook.edu.

THOMAS ROBERTAZZI Prof. Thomas Robertazzi is a Professor at Stony Brook University. He is an IEEE Fellow. He received the PhD from Princeton University and the B.E,E, from the Cooper Union. He has published extensively in areas such as petascale and exascale computing, networking, scheduling, performance evaluation, parallel processing, etc. His email address is thomas.robertazzi@ stonybrook.edu. 